\title{
Influence of Taiji Ball on Health Physical Fitness and Biochemical Indexes of Old People
}

\author{
Jinquan Zeng, Xiaolei Cheng
}

Wuhan City Polytechnic, Wuhan, Hubei Province, China

\begin{abstract}
Keywords: Taiji ball; the elder; health and physical fitness; biochemical index
\end{abstract}
\begin{abstract}
In order to discuss the influence of Taiji Ball on health physical fitness and biochemical indexes of the elder, and to offer a reference frame for the elder to choose the suitable and effective sport project, 17 people aged from 60 to 69 are randomly chosen from a certain activity center. Firstly, to learn the Taiji ball for 20 days, and then practice with relevant music, requiring 1-1.5 hours' collective practice every day and no less than 30mins personally. Results show that the influence of Taiji Ball on health physical fitness and biochemical indexes of the elder is positive and effective. It can also prevent the elder from some relevant disease. It is suitable for the elder to practice the Taiji ball because they can adjust the work-out burden and sport tension according to their own physical situation. What's more, due to the non-confrontation, the practice of Taiji ball can be used as a normal project for the elder to keep healthy.
\end{abstract}

\section{Introduction}

China is a huge country with large population, and also a country rapidly stepping into the aging state. Up to the end of 2008, there are 1.69 hundred million, which occupies $12.79 \%$ of the whole national population. Up to 2100 , the aging level will steadily be $31 \%$. The aging of population will definitely has a great influence on our polity, economy and social environment. Therefore, the party and nation put the public sport development project to a brand new position and conduct the supervision of the national physical quality in 2000, 2005 and 2010. The supervision result shows that the general level of the national physical quality in 2005 is a little higher than which in 2000 . However, the level of body function descent obviously. Among which, the elder between age 60-69 has a large descend range. Currently, the research on the national physical quality mainly concentrates on the group of children and students. As to the elder whose physical function is in recession and physical activity is declining naturally, the relevant research on the health and physical fitness is rather rare.

The theoretical achievement of the health and physical fitness concept, which is brought up aiming to adapt the modern rapid pace and the high efficient social life way, has a positive influence on strengthening the national body quality, promoting social productivity, increasing national comprehensive power. Therefore, it gathers a widely attention and recognition in the field of physical quality research. By contrast, the domestic research on the body adjustment is rare, especially on the aspect of the elder's scientific fitness. The research on the aspect of body quality and function is static while the research on the relevant promotion is rather few. As the consequence, to offer the adequate and effective fitness project for the elder is the current important task for sport researchers.

\section{Research Object and Method}

Research Object. In order to discuss the influence on health physical fitness and biochemical indexes of the elder by practicing Taiji ball, this research randomly chooses 17 healthy people aged from 60 to 69 from a certain activity center for the elder as the object.

Research Method. Literature survey: to collect, read, collate the relevant literature material by the way of internet, books and newspaper, and then offer illustration for this research.

Index chosen: according to the concept of health and physical fitness and the need of this research, 
the experiment chooses some relevant indexes. Body index: height, weight, waistline, hipline, shoulder skin fold, abdomen skin fold, waist skin fold; physiological index: heart rate and blood pressure in peace, vital capacity; body quality index: sit and reach, right hand' grip, reflection, standing on one foot when closing eyes; blood index: rrG, TC, HDL-C, LDL-C.

Experiment design: from 2009.3.9, to learn the Taiji ball for 20 days, to practice with music for 3 months. The practice lasts 1-1.5 hour each day.

Physiological index and body quality data are collected. Before learning Taiji ball, to conduct the test of regular physiological index and body quality (the date is 2009.3.7). After 3 months' practice (the date is 2009.7.1), to conduct the 2nd test.

Blood samples are collected. To collect the blood sample with the patients' empty stomach in the morning and then analyze the physiological data. This collection should also be conducted twice with the same time as 1.2.2.3.

Test tool. Test machine for body quality (height, weight, vital capacity, sit and reach, grip, all transported by wireless data) produced by Beijing Huaxia Huihai technology limited company; Finland s610 heart rate text machine; electronic step test machine, ;stop watch(to reserve), skin fold measuring apparatus; electronic measuring apparatus for standing on one foot while closing eyes; electronic reaction measuring apparatus; electronic grip machine; to entrust Zhumadian center hospital to test and analyze the blood sample.

Statistic analysis: applying Excel 2003 and SPSS17.0 to handle and analyze the data material. The statistic method is $\mathrm{T}$ Test.

\section{Result and Analysis}

The elder citizens practice Taiji ball to effect their body shape 1-1.5h every day. The test after 3 months affirms that the practicing can reduce BMI, which is in accordance with the foreign research. The systematic aerobics can change the body metabolism, adjust and improve fat metabolism, avoid excessive fat accumulation, and then prevent and control the happening and development of obesity. Taiji ball practicing is a kind of useful aerobics. WHR can be widely used to reflect the distribution of body fat. If fat stores largely in chest and abdomen, it will easily cause some disease related to obesity, such as CVD and diabetes. If WHR increases, the body quality will decrease. After 3months' Taiji ball practicing, the male WHR decreases to $0.87 \pm 0.03$ form $0.90 \pm 0.03$, which is very remarkable in diversity $(\mathrm{P}<0.05)$; the female WHR decreases to $0.80 \pm 0.04$ form $0.85 \pm 0.04$, which is very remarkable in diversity too $(\mathrm{P}<0.01)$. Compared with the skin fold thickness in and after 3 months, the influence on the decrease of subjects by practicing Taiji ball is remarkable $(\mathrm{P}<0.01)$, which is related to the abdomen rotation in Taiji ball practicing. Muscle in abdomen will consume a large scale of fat and then decrease the excessive fat in, finally the weight can be controlled.

The influence on the elders' physical index by Taiji ball practicing. Massive researches in both domestic and abroad sporting and medical science reveal that there is a close link between a person's athletic ability and cardio-pulmonary function, as well as health condition. The athletic ability can reflect the body's functional reservation and operation. The Taiji ball practicing has many posts, such as holding heads high and twisting, which calls for high requirement for body's breath system. Inhaling requires that the chest must expand totally, making much more lung bubble to expand, and then strength the power of breathing muscle, enlarge the chest expanding realm, finally amplify vital capacity. The long-term Taiji ball practicing can also promote blood circulation, open much more blood capillary, and then decrease the blood vessel's surrounding resistance, smooth the blood vessel, keep the blood vessel's elasticity, decrease systolic pressure, finally lower blood pressure. The experiment data verify the above opinion primely, complying with Fu Xiao, Zhang Shang's research result. The long-term aerobic exercise can improve the elders' strength of breath muscle effectively, increase breath efficiency and vital capacity, and finally decrease blood pressure.

The influence on the elders' body quality by Taiji ball practicing. When old age comes, joint capsule, ligament, muscle tendon etc. will become maturing gradually, and then affect the body's stretch. Taiji ball practicing includes many rotation posts with waist as axis, such as turn-over, twist, and side-rotation, which can exercise waist muscle well and promote waist joint's flexibility. Data 
shows that Taiji ball practicing can improve the stretch of every ligament, muscle tandon and skin, making the elders' joints much more flexible $(\mathrm{P}<0.05)$.

Some researches deem that the aerobic exercise can promote the blooding supply of nerve muscle, decelerate the blood vessel's maturing degree and improve the function of body balance. Taiji ball practicing is centered in circle. Its rotation posts such as turn-over, twist, side-rotation etc. will benefit to the elders' balance ability. The change data of the subjects' standing on one foot while closing eyes project proves that the long-term Taiji ball practicing can improve the elders' balance ability.

Grip level can reflect the senile change degree of the elders' body. The body of the crowd seldom practicing gets weak when their age gets older. Reaction then relates to the developmental and sentile period of body's centre nerve system. Generally, reaction period gradually shortens from childhood to adolescence, and then increase afterwards and becomes longer after 60. Since Taiji ball practicing has many rotation posts in upper arms, which requires practicers' sight following with the ball, gesture smooth and coordinate. Keeping practicing frequently will do good to increase the function of nerve system. The experiential data shows that after 3months' Taiji ball practicing, the male reaction duration decreases from $0.91 \pm 0.20$ to $0.68 \pm 0.13$, while female reaction duration decreases from $1.02 \pm 0.32$ to $0.74 \pm 0.20$.It is remarkable $(\mathrm{P}<0.05)$ and complies with Chen yuefeng $(1994)$ 's research result. This test reveals that adequate Taiji ball practicing can boost the body's reaction speed, add nerve system's flexibility, and also prevent the elder from senite dementia.

Elders' blood biochemical index is also influenced by practicing Taiji ball. TC is an important part of the body's oganic cell. A long-term increase of cholesterin will accumulate in the blood vessel, making the hyperplasia of the artery smooth skin cell and fiber cell, add the danger of the artery sticky and the disease of hypertension and cardiovascular and cerebrovascular. Some researchers find that the acute exercise for one time has little effect on the blood TC' concentration, while a long-term exercise can cuuse the concentration to decrease. Athletical exercise can enhance the hypeerlipoprotenemia's activity, promote the blood TG's hydrolysis, accelerate the bone muscle's usage on fatty acid, and then prompt TG deliver from liver.

As a kind of aerobic exercise of low physical burden and tension, Taiji ball practicing can increase the hypeerlipoprotenemia's activity, accelerate fat's oxidation, make the full use of the excessive fat under the circumstance of adequate oxygen, and then decrease the concentration of TC and LDL-C, add the concentration of HDL, reduce the accumulation of fat in blood vessel, accelerate the TG's hydrolysis and the bone muscle's usage on fatty acid, strengthen the sensibility of the surrounding part on insulin, decline insulin's resistance.

After 3 months' Taiji ball practicing, the male TC changes obviously ( $\mathrm{P}<0.01)$, male HDL-C changes obviously, too (P\%0.05); the female index changes obviously (P\%0.01), LDL-C obviously, too (P\%0.01). The practice of Taiji ball can increase the elders' HDL, reduce LDL, which can prevent the elder from getting the disease of artery sticky and the disease of hypertension and cardiovascular and cerebrovascular. It indicates that Taiji ball practicing is suitable for the elder.

\section{Conclusion}

Taiji ball practicing combined with the essence of Taiji and modern ball, centered in the axie of waist, emphasizes the coordination of hands, eyes, brain and all fours and do good for the elders' neck, shoulder waist and legs. As an aerobic sport, Taiji ball practicing can adjust both big and small muscle group and joints, which can enhance the elders' flexibility of muscle strength and joints, control weight, reduce blood fat, and then prevent the disease of cardiovascular and cerebrovascular.

\section{References}

[1] Clinical exercise testing. WEISMANI M, ZEBALLOS RJ. Clinics in Chest Medicine. 2001

[2] Exerciseintreating hypertension. Jirayos C, David T. Physician and Sportsmedicine. 2002

[3] Studies on the plasma lipid profiles, and LCAT and CETP activities according to Hyperlipoproteinemia phenotypes (HLP). Lee M, Kim J Q, Kim J, et al. Atherosclerosis. 2001 
[4] An exploratory randomised controlledtrial of a support programme for carers of patients with apsychosis [J]. G. Szmukler, E. Kuipers, J. Joyce, T. Harris, M. Leese, W. Maphosa, E. Staples. Social Psychiatry and Psychiatric Epidemiology. 2003 (8) 